Mens

revue d'histoire intellectuelle de l'Amérique française

\title{
Alan Mendelson. Exiles from Nowhere: The Jews and the Canadian Elite, Montréal, Robin Brass Studio, 2008, 416 p.
}

\section{Jean-Philippe Croteau}

Volume 11, numéro 1, automne 2010

URI : https://id.erudit.org/iderudit/1023343ar

DOI : https://doi.org/10.7202/1023343ar

Aller au sommaire du numéro

Éditeur(s)

Centre de recherche en civilisation canadienne-française

ISSN

1492-8647 (imprimé)

1927-9299 (numérique)

Découvrir la revue

Citer ce compte rendu

Croteau, J.-P. (2010). Compte rendu de [Alan Mendelson. Exiles from Nowhere:

The Jews and the Canadian Elite, Montréal, Robin Brass Studio, 2008, 416 p.]

Mens, 11(1), 113-117. https://doi.org/10.7202/1023343ar d'utilisation que vous pouvez consulter en ligne.

https://apropos.erudit.org/fr/usagers/politique-dutilisation/ 
de théoriciens non canadiens du phénomène technologique et les penseurs canadiens. L'état de la réflexion sur la technologie dans d'autres contextes nationaux est marginalement relaté. Une exploration plus rigoureuse de ces contextes aurait permis de saisir les points d'inflexion de "l'impératif technologique " et de mieux comprendre comment ces auteurs, dispersés dans le temps et dans la pratique, finissent par se pencher de la même façon sur un même objet. En conséquence, il est difficile d'évaluer dans les réflexions ici recensées la part d'originalité et la part d'emprunt à une pensée occidentale qui a composé au cours de la même période avec les mêmes développements technologiques, sans égard aux cultures des sociétés d'accueil. Dans quel cas verrions-nous poindre encore une fois un déterminisme technologique.

\section{- Stéphane Castonguay Centre interuniversitaire de recherche sur la science et la technologie Université du Québec à Trois-Rivières}

\section{Alan Mendelson. Exiles from Nowhere: The Jews and the Canadian Elite, Montréal, Robin Brass Studio, 2008, 416 p.}

Au Canada, aux États-Unis et en Europe, l'histoire de l'antisémitisme ne cesse d'éveiller les intérêts et de soulever les débats. Au Canada, cette question prend beaucoup d'importance sans doute en raison des croyances fermement établies parmi le grand public que le pays aurait entretenu une tradition d'ouverture et de tolérance tout au long de son histoire. Néanmoins, rappelons que depuis la polémique suscitée par la thèse d'Esther Delisle, dans les années 1990, l'historiographie s'est enrichie de travaux qui brossent un portrait plus nuancé et plus complexe de l'antisémitisme, de la fin du XIX ${ }^{\mathrm{e}}$ siècle jusqu'au lendemain de la Seconde Guerre mondiale. 
Or la plupart des travaux des historiens continuent d'être cloisonnés entre les multiples interprétations qui varient en grande partie selon que l'on s'intéresse à l'antisémitisme canadien-anglais ou canadien-français. Ainsi, l'antisémitisme canadien-anglais apparait, pour les historiens, comme un phénomène périphérique, marginal et épisodique, atténué en grande partie par des traditions libérales et démocratiques fortement enracinées dans la culture britannique et protestante. De fait, les historiens se sont surtout attachés à présenter les manifestations et les modes d'expression politique et sociale de l'antisémitisme canadien-anglais plutôt que ses fondements idéologiques. Le regard posé sur l'antisémitisme canadien-français est basé, quant à lui, sur une approche essentialiste qui tend à reconnaître le caractère central de ce courant idéologique au sein de la société canadienne-française dite "traditionaliste ", qui le rend indissociable et parfois presque " naturel », comme s'il allait de soi.

Le présent ouvrage propose d'embrasser un nouveau champ d'analyse en s'intéressant à l'antisémitisme des élites canadiennesanglaises et, surtout, à ses postulats idéologiques. Cette approche prend ses distances avec la tradition historiographique sur l'antisémitisme canadien-anglais qui, à l'inverse de l'historiographie sur l'antisémitisme canadien-français, avait préféré mettre de côté les fondements et les racines idéologiques de ce courant de pensée pour s'intéresser davantage aux pratiques sociales et politiques. L'auteur, Alan Mendelson, tente de montrer que l'antisémitisme, étendu à un segment important des élites canadiennes-anglaises, s'exprimait surtout au nom du "Christian Triumphalism", une conception philosophique et théologique qui croit en la supériorité et en l'hégémonie du christianisme sur le judaïsme. Pour démontrer sa thèse, l'auteur a étudié des figures politiques et intellectuelles notoires de l'histoire canadienne, en particulier les membres de la famille Grant, dont le plus éminent est George Parkin Grant, auteur de Lament for a Nation.

L'auteur tente de reconstituer la pensée de ces personnalités politiques et intellectuelles en consultant leurs publications, livres, 
pamphlets, articles, discours, et, en particulier, leur correspondance privée et leurs notes personnelles. L'importance accordée à ces dernières sources lève le voile sur des aspects peu connus de leur pensée, qui n'étaient pas toujours exprimés sur la place publique et qui étaient plutôt réservés à des cercles d'amis et d'intimes. Grâce à ces sources privilégiées, l'auteur recrée non seulement le réseau des relations sociales des personnalités étudiées, mais il établit aussi leur filiation intellectuelle avec leurs mentors et leurs maîtres à penser.

Cette approche a l'avantage de jeter un regard très fouillé et très détaillé sur la pensée de ces figures intellectuelles qui révèle beaucoup sur leur vie publique. Ainsi, le livre est riche en informations sur la relation d'amitié entre Henri Bourassa et Goldwin Smith, un libéral admiré, mais aussi un antisémite notoire. Il rend compte aussi de la méfiance de Mackenzie King envers les Juifs à travers ses écrits personnels, de ses études jusqu'à son retrait de la vie publique. Ses impressions sur Hitler, consignées dans son journal intime lors de son entretien avec ce dernier, nous renseignent sur l'admiration du premier ministre canadien pour le chancelier allemand, sa complaisance pour le régime nazi et le peu de cas qu'il faisait des persécutions raciales envers les Juifs. Le portrait intellectuel que brosse l'auteur de George Monro Grant, recteur de l'Université Queen's, nous montre un exemple concret de cette croyance en la suprématie des religions chrétiennes (voire le protestantisme), dont la mission était de christianiser le monde.

L'aspect le plus intéressant, peu examiné par les historiens à ce jour, est la germanophilie et l'antisémitisme des élites anglo-canadiennes dissimulés derrière un pacifisme militant dans le contexte des tensions internationales des années 1930. Ainsi, Vincent Massey, haut-commissaire canadien au Royaume-Uni, entretenait des rapports privilégiés avec le couple Astor, aristocrates britanniques bien connus pour leurs sympathies envers l'Allemagne nazie et qui soutenaient la politique d'apaisement de Chamberlain vis-à-vis de Hitler. Dans quelle mesure ce pacifisme, puisé en partie dans la germanophilie et l'antisémitisme avoués d'une partie de l'élite britannique 
et anglo-canadienne, a-t-il pu dicter les orientations de la politique étrangère canadienne pendant l'entre-deux-guerres caractérisées par l'isolationnisme et la neutralité ? Cette piste de recherche mériterait d'être davantage approfondie.

Cependant, l'approche méticuleuse de Mendelson, qui décortique la pensée de ces intellectuels dans les moindres détails, comporte nombre de faiblesses. L'auteur néglige le contexte social et politique dans lequel évoluent les personnalités choisies. Ainsi, le lecteur ne sait rien des facteurs qui ont pu produire, puis propager le courant de pensée antisémite à l'intérieur des cénacles sociopolitiques fréquentés par les élites anglo-canadiennes. Dans cet ouvrage, il n'y a aucune tentative d'explication. L'antisémitisme semble uniquement généré par les individus. C'est avant tout une analyse biographique fort descriptive, détachée de la réalité sociologique de l'époque étudiée. De surcroît, des courants idéologiques comme l'impérialisme britannique ou le darwinisme social, qui ont eu une certaine influence sur l'antisémitisme au Canada, sont traités en surface par l'auteur. D'autres lacunes conceptuelles peuvent être relevées. Mendelson ne définit pas l'antisémitisme, s'attarde peu à ses conditions d'émergence et ne cherche pas à distinguer l'antisémitisme, doctrine raciale et sociale qui naît à la fin du XIX ${ }^{\mathrm{e}}$ siècle, de l'antijudaïsme, un mouvement de pensée à caractère religieux, qui prend racine au Moyen Âge. Pourtant, les intellectuels analysés puisent abondamment dans ces deux registres lorsqu'ils abordent la question juive.

L'aspect qui pose le plus problème reste le choix des figures intellectuelles que Mendelson étudie. L'examen de la pensée antisémite entrepris par l'auteur se limite surtout aux membres d'une même famille, associés par des alliances matrimoniales. À l'exception de la première partie du livre, qui se penche sur la pensée de Goldwin Smith, d'Henri Bourassa - qui n'est pas un membre de l'élite anglocanadienne, soit dit en passant - et de Mackenzie King, tous les personnages étudiés entretiennent un lien de parenté avec la famille Grant. Ce portrait réducteur des élites anglo-canadiennes se limite, en fait, à une "dynastie intellectuelle ». Il est à se demander quelle 
est la véritable représentativité des membres de cette famille, alors que Mendelson tend à généraliser leurs opinions sur les Juifs à une partie importante des élites anglo-canadiennes.

Il apparaît plutôt que l'ouvrage a servi de prétexte pour aborder l'antisémitisme de George Parkin Grant, icône intellectuelle au Canada anglais, que l'auteur a par ailleurs connu personnellement. En effet, la partie couvrant la trajectoire intellectuelle de George Parkin Grant constitue près de la moitié du livre. Il reste que, sur cet aspect, Mendelson parvient à dresser les origines intellectuelles de la pensée de Grant qui auraient nourri, selon lui, son antisémitisme en passant en revue méthodiquement ses sources d'inspiration (Arnold Toynbee, Martin Heidegger, Louis-Ferdinand Céline et Simone Weil). Il ressort de sa démonstration qu'il existait dans la pensée de Grant un rapport subtil entre une conception qui prétendait à la supériorité du christianisme et une forme de préjugé antisémite insidieux. Il apparaît cependant que, contrairement à Goldwin Smith, qui fait l'objet du premier chapitre de cet ouvrage, Grant n'a pas fait de l'antisémitisme l'un des pôles principaux de sa pensée ni l'un de ses chevaux de bataille dans ses interventions publiques.

Tout bien considéré, il faut rappeler le caractère novateur et même pionnier de cette étude, l'une des premières à accorder autant d'attention aux postulats idéologiques de l'antisémitisme des élites anglo-canadiennes et qui vient entrouvrir une porte dans un champ de recherche peu exploré. Il est à espérer que l'on n'en reste pas là et que l'on puisse assister à la publication d'une étude qui inclurait dans un même canevas l'univers mental et idéologique de l'antisémitisme des élites anglo-canadiennes et ses répercussions sur les manifestations et les modes d'expression politique, les pratiques sociales et les rapports sociaux. Bref, rétablir le chaînon manquant qui, actuellement, fait cruellement défaut à une explication sociohistorique de l'antisémitisme au Canada anglais, lequel a perduré de la fin du XIX ${ }^{e}$ siècle jusqu'au lendemain de la Seconde Guerre mondiale. 